\title{
Eight-IncRNA signature of cervical cancer were identified by integrating DNA methylation, copy number variation and transcriptome data
}

\author{
Qihang Zhong ${ }^{1,2+}{ }^{+}$, Minzhen $\mathrm{Lu}^{2,3,4,5+}$, Wanqiong Yuann ${ }^{6,7 \dagger}$, Yueyi Cui ${ }^{2}$, Hanqiang Ouyang ${ }^{6}$, Yong Fan ${ }^{8}$, \\ Zhaohui Wang ${ }^{9}$, Congying W W $^{1 *}$, Jie Qiao ${ }^{2,3,4,5^{*}}$ and Jing Hang ${ }^{2,3,4,5^{*}}$
}

\begin{abstract}
Background: Copy number variation (CNV) suggests genetic changes in malignant tumors. Abnormal expressions of long non-coding RNAs (IncRNAs) resulted from genomic and epigenetic abnormalities play a driving role in tumorigenesis of cervical cancer. However, the role of IncRNAs-related CNV in cervical cancer remained largely unclear.

Methods: The data of messenger RNAs (mRNAs), DNA methylation, and DNA copy number were collected from 292 cervical cancer specimens. The prognosis-related subtypes of cervical cancer were determined by multi-omics integration analysis, and protein-coding genes (PCGs) and IncRNAs with subtype-specific expressions were identified. The CNV pattern of the subtype-specific IncRNAs was analyzed to identify the subtype-specific IncRNAs. A prognostic risk model based on IncRNAs was established by least absolute shrinkage and selection operator (LASSO).

Results: Multi-omics integration analysis identified three molecular subtypes incorporating 617 differentially expressed IncRNAs and 1395 differentially expressed PCGs. The 617 IncRNAs were found to intersect with diseaserelated IncRNAs. Functional enrichment showed that 617 IncRNAs were mainly involved in tumor metabolism, immunity and other pathways, such as p53 and CAMP signaling pathways, which are closely related to the development of cervical cancer. Finally, according to CNV pattern consistent with differential expression analysis, we established a IncRNAs-based signature consisted of 8 IncRNAs, namely, RUSC1-AS1, LINC01990, LINC01411, LINC02099, H19, LINC00452, ADPGK-AS1, C1 QTNF1-AS1. The interaction of the 8 IncRNAs showed a significantly poor prognosis of cervical cancer patients, which has also been verified in an independent dataset.
\end{abstract}

Conclusion: Our study expanded the network of CNVs and improved the understanding on the regulatory network of IncRNAs in cervical cancer, providing novel biomarkers for the prognosis management of cervical cancer patients.

Keywords: Copy number variation, Multi-omics integration analysis, IncRNA signature, Cervical cancer

*Correspondence: congyingwu@hsc.pku.edu.cn; jie.qiao@263.net; hangjbysy@163.com

${ }^{\dagger}$ Qihang Zhong, Minzhen Lu, Wanqiong Yuan: Equal contribution ${ }^{1}$ Institute of Systems Biomedicine, School of Basic Medical Sciences, Peking University Health Science Center, Peking University, HaiDian District, No. 38 XueYuan Road, Beijing 100191, China ${ }^{2}$ Center for Reproductive Medicine, Department of Obstetrics and Gynecology, Peking University Third Hospital, HaiDian District, No. 49 North HuaYuan Road, Beijing 100191, China

Full list of author information is available at the end of the article

\section{Background}

Cervical cancer is one of the most frequently diagnosed cancers and a leading cause of cancer deaths to women [1]. Annually there are 500,000 newly diagnosed cases of cervical cancer and 300,000 deaths, and $80 \%$ of all the cervical cancer cases occur in developing regions [2]. Human papillomavirus (HPV) infection, particularly HPV 16 and HPV 18, and immunosuppression, smoking, pregnancy history and long-term use of

c) The Author(s) 2021. This article is licensed under a Creative Commons Attribution 4.0 International License, which permits use, sharing, adaptation, distribution and reproduction in any medium or format, as long as you give appropriate credit to the original author(s) and the source, provide a link to the Creative Commons licence, and indicate if changes were made. The images or other third party material in this article are included in the article's Creative Commons licence, unless indicated otherwise in a credit line to the material. If material is not included in the article's Creative Commons licence and your intended use is not permitted by statutory regulation or exceeds the permitted use, you will need to obtain permission directly from the copyright holder. To view a copy of this licence, visit http://creativeco mmons.org/licenses/by/4.0/. The Creative Commons Public Domain Dedication waiver (http://creativecommons.org/publicdomain/ zero/1.0/) applies to the data made available in this article, unless otherwise stated in a credit line to the data. 
oral contraceptives are the most critical risk factors for developing cervical cancer [3]. The techniques of cervical cancer screening such as the papanicolaou and HPV detection have been greatly improved in the past decades [4]. The infection of high-risk HPV does not necessarily lead to cervical cancer, suggesting that HPV infection is a principal but not a decisive cause of cervical cancer [5]. Study found that cervical cancer could develop independently by genetic changes with altered expressions of oncogenes or tumor suppressor genes or together with HPV infection [6]. The five-year survival rate for cervical cancer patients with early detection of cervical cancer is 92\% [4], but the chance drops sharply for patients with tumor spreading to surrounding tissues or other distant organs. Therefore, the early detection of cervical cancer has a strong significance in cervical cancer intervention and treatment.

Copy number variation (CNV) is defined as a variation in genetic structure, usually between increase or decrease of $1 \mathrm{kB}-3 \mathrm{Mb}$ at the copy number of a genome fragment [7]. Copy number amplification or deletion in cancer genome often leads to the inactivation of tumor suppressor genes or the expressions of oncogenes that have important effects on cell functions, including cell adhesion and recognition [8]. For example, the increase in the copy number of PD-L1 gene has been found to be related to the anti-PD-1/PD-L1 treatment of locally advanced cervical cancer [9]. HPV16 and HPV18 genome copies are associated with the grade of cervical lesions [10]. CNV not only refers to tumor biology, but also have effects on some complex immune diseases. Patients with copy number abnormalities of CCL3L1 are more susceptible to HIV/acquired immunodeficiency syndrome (AIDS) [11]. CNV of human Fcgr3 gene is determinant of glomerulonephritis [12]. At present, a large number of studies focused on messenger RNAs (mRNAs) and investigated the phenotypic changes and tumor progression caused by CNVs, while only a few studies were conducted to analyze the regulatory relationship between CNVs and non-coding RNAs, especially long non-coding RNAs (lncRNAs).

LncRNAs are defined as RNA transcripts with more than 200 base pairs in length and are a major class of ncRNA [13]. Abnormally expressed lncRNAs are closely related to complex human diseases, especially in tumors [14]. Dysfunctions of lncRNAs contribute to the development, progression and metastasis of cancers [15]. For example, lncRNA ARAP1-AS1 promotes the translation of the proto-oncogene c-Myc by isolating PSF/PTB dimers in cervical cancer, thereby promoting tumorigenesis and metastasis [16]. LncRNA NKILA inhibits proliferation and promotes the apoptosis of cervical squamous cells by down-regulating miRNA-21 expression [17].
LncRNA SBF2-AS1 enhances cervical cancer progression through regulating miR-361-5p/FOXM1 axis [18]. Expression profile shows highly abnormal expressions of lncRNAs in cancer, suggesting that lncRNAs could serve as a biomarker for predicting clinical outcomes [19].

High-throughput technologies allow histological studies to interrogate thousands of manufacturers with similar biochemical properties (e.g., RNA transcriptomes). Monolayer "histology" provides only limited insight into the biological mechanisms of diseases. In genomewide association studies, although a great number of single-nucleotide polymorphisms have been identified for complex diseases and traits, the functional implications and mechanisms of the loci of interest remain largely unknown. In addition, genomic variation alone cannot fully explain changes in disease risk over a lifetime; DNA, RNA, proteins, and metabolites often play complementary roles and perform certain biological functions together. Such complementary effects and synergies between genomic layers in a life course can only be obtained through comprehensive studies of multiple molecular layers [20].

In this study, based on mRNA expressions, DNA methylation, and DNA copy number, we identified three molecular subtypes related to the prognosis of patients with cervical cancer. Differentially expressed mRNAs and lncRNAs in the three molecular subtypes were analyzed and examined to analyze the functions of co-expressed lncRNAs. Although CNVs play important role in transcriptional regulation, it was unclear whether $\mathrm{CNV}$ is systematically related to the expressions of lncRNAs in cervical cancer. By analyzing the copy number profile of lncRNAs across the genome, we carefully examined these abnormal lncRNAs induced by copy number amplification or deletion. In addition, Kaplan-Meier (KM) survival analysis was conducted to assess the prognostic performance of the lncRNAs with copy number abnormalities. Finally, a prognostic model was established to predict the survival of cervical cancer patients. This study aimed to identify CNV-related lncRNAs that can better predict cervical cancer prognosis.

\section{Methods}

\section{Data download and processing}

Methylation data, RNA-seq data, CNV, whole exome sequencing (WES) mutation data and sample followup information of Illumina Infinium $450 \mathrm{k}$ Human DNA methylation Beadchip v1.2 platform for cervical cancer were downloaded from The Cancer Genome Atlas (TCGA) database (https://tcga-data.nci.nih.gov/) [21]. All the samples were collected before the first treatment. RNA-seq counts were converted into TPM (TranscriptsPerKilobase of exonmodel per Million mapped 
reads) expression profile data. Finally, expression profile data sets incorporating 304 cancer tissue samples and 3 para-cancer samples were obtained. According to the GeneCode v33 GTF [22], the expression profiles of 14,851 sense-intronic, sense-overlapping, antisense, processed-transcript, or primer-overlapping lncRNAs were acquired when gene type was defined as the lncRNA. When gene type was defined as protein-coding genes (PCGs), the expression profiles of 19,611 PCGs were obtained. For methylation data, CpG probes with the presence of NA expression in each sample were removed. At the same time, according to the cross-reactive site provided by Chen et al. [23], the CpG sites with crossreactive in the genomes or unstable genome methylation sites were all removed, that is, the CpGs and single nucleotide sites on the sex chromosome were removed. In this way, a total of 372,137 CpGs sites were finally obtained. A total of 304 samples of copy number variation data were acquired after the removal of germline CNV data. Single nucleotide mutation data processed by MuTect software and clinical follow-up information of 307 cervical cancer samples were downloaded. A total of 292 primary tumor samples before the first treatment with complete data of RNA-seq, SNV, and methylation were detected for multi-omics clustering analysis. Meanwhile, standardized expression profile data GSE19711 containing 300 cervical cancer samples was download from the Gene Expression Omnibus (GEO) database (http://www.ncbi.nlm.nih.gov/ geo/), with Illumina HumanHT-12 WG-DASL V4.0 R2 expression beadchip platform as an external validation. Sample information in TCGA data and GEO dataset are shown in Table 1.

Table 1 Clinical features of the data set

\begin{tabular}{|c|c|c|c|c|c|}
\hline \multirow[t]{2}{*}{ Characteristics } & \multicolumn{4}{|l|}{ TCGA-Set } & \multirow[t]{2}{*}{ GSE44001 $(N=300)$} \\
\hline & Common Samples & RNA-seq $(N=304)$ & Methylation $(\mathrm{N}=304)$ & CNV $(N=340)$ & \\
\hline \multicolumn{6}{|l|}{ Survival event } \\
\hline Dead & 67 & 72 & 71 & 67 & 38 \\
\hline Live & 225 & 232 & 233 & 235 & 262 \\
\hline \multicolumn{6}{|l|}{ Pathological_T } \\
\hline $\mathrm{T} 1$ & 134 & 140 & 140 & 139 & NA \\
\hline $\mathrm{T} 2$ & 69 & 70 & 71 & 71 & NA \\
\hline T3 & 20 & 21 & 20 & 21 & NA \\
\hline $\mathrm{T} 4$ & 8 & 10 & 10 & 8 & NA \\
\hline \multicolumn{6}{|l|}{ Pathological_N } \\
\hline No & 127 & 133 & 133 & 133 & NA \\
\hline $\mathrm{N} 1$ & 56 & 61 & 60 & 58 & NA \\
\hline N2 & 0 & 0 & 0 & 0 & NA \\
\hline N3 & 0 & 0 & 0 & 0 & NA \\
\hline \multicolumn{6}{|l|}{ Pathological_M } \\
\hline MO & 108 & 114 & 116 & 111 & NA \\
\hline M1 & 10 & 11 & 10 & 10 & NA \\
\hline \multicolumn{6}{|c|}{ Pathological_Stage } \\
\hline Stage I & 40 & 44 & 44 & 42 & 258 \\
\hline Stage II & 55 & 56 & 57 & 57 & 42 \\
\hline Stage III & 45 & 46 & 45 & 46 & 0 \\
\hline Stage IV & 19 & 22 & 21 & 19 & 0 \\
\hline \multicolumn{6}{|c|}{ Pathological_Grade } \\
\hline G1 & 18 & 19 & 18 & 18 & NA \\
\hline G2 & 128 & 134 & 135 & 133 & NA \\
\hline G3 & 113 & 118 & 118 & 118 & NA \\
\hline G4 & 1 & 1 & 1 & 1 & NA \\
\hline \multicolumn{6}{|l|}{ Age } \\
\hline$>=60$ & 56 & 59 & 59 & 56 & NA \\
\hline$<60$ & 236 & 245 & 245 & 246 & NA \\
\hline
\end{tabular}




\section{Prognostic molecular subtype identification based on multi-omics}

The iClusterPlus [24], which is developed for comprehensive cluster analysis of multiple types of genomic data, is an enhanced version of iCluster. In this study, iClusterPlus was used to identify molecular subtypes based on DNA methylation, CNVs and transcriptome data. Specifically, we first analyzed the effects of PCGs, CNVs, and methylation on the prognosis of cervical cancer. Then the prognostic-related characteristics of DNA methylation, CNVs and transcriptome were examined by establishing Univariate COX proportional risk regression model. For potentially relevant features, the significance threshold was set as $\mathrm{p}<0.05$. Next, DNA methylation, CNVs and transcriptome samples were integrated to extract the data corresponding to the features related to the cancer prognosis. The $\mathrm{R}$ software package iClusterPlus [25] in $\mathrm{R}$ software package was further used for cluster analysis, The copy number data were segmented using the CBS algorithm21. The segment means were used as the input for integration to reduce the noise level, and the different histological data were further z-transformed and clustered using the K-means clustering algorithm, with the classification results of $k=2-10$. Euclidean distance was used to assesses the distance of difference between samples. The classification results with the least difference within the group and the greatest difference among the groups were selected and employed to determine the molecular subtypes of cervical cancer prognosis.

\section{Subtype identification based on differentially expressed IncRNAs and PCGs}

We used the $R$ package DESeq2 [26] to identify lncRNAs and PCGs with subtype differences. Firstly, after removing the genes with an average count of $<5$ in the expression profile, the differences of each subtype were compared. Other samples outside the subtype were taken as the control group, with the threshold of foldchange greater than twice and FDR $<0.05$. Furthermore, the absolute value of the difference multiple was used as the rank order, and Gene Set Enrichment Analysis (GSEA) was applied to detect the distribution of DElncRNAs. The ncRNAs closely related to cervical cancer were downloaded from LncRNADisease [27], with Lnc2Cancer [28] database serving as the background for the comparison of the relationship between de-lncRNAs and cervical cancer.

\section{Identification of enriched IncRNA modules by weighted gene co-expression network analysis}

Weighted gene co-expression network analysis (WGCNA) package [29] in $R$ was used to construct a scale-free coexpression network for the differentially methylation sites
(DMPs). Pearson's correlation matrices and average-linkage method were both conducted for differential expression (DE)-PCGs/lncRNAs. $\beta$ was a soft-thresholding parameter that emphasizes strong association between PCGs and penalized weak association. Then, the adjacency was converted into a topological overlap matrix (TOM), which was defined by the sum of its adjacency with all other DEPCGs/lncRNAs for network DE- PCGs/lncRNAs ration, and the corresponding dissimilarity (1-TOM) was calculated. $p<0.05$ was set as the threshold to identify the modules with significant enrichment in DE-lncRNAs. Finally, based on the genes in the DE-lncRNAs enrichment module, $\mathrm{R}$ package clusterprofiler was used to perform Kyoto Encyclopedia of Genes and Genomes (KEGG) pathway enrichment analysis.

\section{Genomic copy number anomalies and their relationship to IncRNAs were analyzed by GISTIC algorithm}

Genes targeted by somatic cell copy number change (SCNA) play an important role in tumorigenesis and cancer therapy. Here, we used GISTIC2.0 [30] software to define CNVs extracted from of all the genes in the 292 cervical cancer samples were related to lncRNAs. Copy number $>1$ or $<-1$ was defined as copy number amplification and deletion, respectively. According to $\mathrm{FDR}<0.05$, the boundaries of the regions with peak amplification or deletion limits identified by GISTIC 2.0 were determined with at least $95 \%$ confidence to include the target gene/lncRNA(s). Samples with lncRNA expression profiles were selected, lncRNA expression profiles and copy numbers were calculated by Spearman's rank correlation coefficient, and random differences in the distribution of correlation coefficients were compared. Finally, the lncRNAs with CNV in more than $25 \%$ of the samples were identified, and their expression differences were compared in copy amplification and copy deletion samples.

\section{Identification of IncRNA prognostic markers with abnormal copy number and establishment of gene signature based on IncRNAs}

We systematically analyzed the CNV of these de-lncRNAs on the basis of molecular subtypes, and selected lncRNAs with a proportion of abnormal copy number higher than $15 \%$. Univariate cox was used to analyze the relationship between these IncRNAs and overall survival (OS). The threshold was defined as $\mathrm{p}<0.01$ to screen lncRNAs significantly related to the prognosis of cervical cancer. In addition, least absolute shrinkage and selection operator (LASSO) Cox was used for analyzing the expression profiles of these lncRNAs for feature selection [31]. The tenfold cross-validation was used to construct the model. Finally, the Multivariate Cox survival analysis was performed, and 
the lncRNAs with minimal Akaike information criterion (AIC) value was considered as the final prognostic markers to establish the risk score model:

$$
\text { RiskScore }=\sum_{k=1}^{n} \operatorname{Exp}_{k} * e^{H R}{ }_{k}
$$

where $\mathrm{N}$ is the number of prognostic $\operatorname{lncRNAs}, \operatorname{Exp}_{k}$ is the expression value of prognostic lncRNAs, and $e^{H R}{ }_{k}$ is the estimated regression coefficient of IncRNAs in the multivariate Cox regression analysis.

\section{Functional enrichment analysis}

GSVA [32] was performed using the $\mathrm{R}$ package on $\mathrm{C} 2$ Canonical pathway gene set collection that contained 1320 gene sets obtained from MsigDB database. The enrichment scores of each sample in these gene sets were analyzed using single sample GSEA, and the association between the enrichment scores of each gene sets and RiskScore was further calculated by Spearman's rank correlation coefficient. KEGG pathway with the absolute correlation coefficient greater than 0.4 and $F D R<0.01$ was selected.

\section{Performance comparison of the IncRNA signatures}

To determine the independence of the lncRNA signature, we performed single-factor and multi-factor Cox regression to analyze the relationships among T, N, M stage, age and risk score and prognosis. Furthermore, we compared the newly established lncRNA signatures with four recently reported prognostic risk models, which were 4-lncRNAs signature by sun et al. [33], 10-lncRNA signature by Shen et al. [34], 9-IncRNAs signature by Mao et al. [35], and 6-lncRNA signature by Luo et al. [36]. To ensure the model comparability, we calculated the risk score of each cervical cancer sample in the TCGA dataset using the same method based on the corresponding genes in these 4 models. Moreover, the receiver operating characteristic (ROC) of each model was determined and the risk score was calculated based on the median risk score. The samples were divided into Risk- $\mathrm{H}$ and Risk- $\mathrm{L}$ groups, and the prognostic differences between the two groups of samples were calculated. Furthermore, we compared these four models with restricted mean survival, concordance index (C-index), and decision curve analysis (DCA) of the lncRNA signature.

\section{Other statistical descriptions}

Except for special instructions, the normality of variables was examined by Shapiro-Wilk normality test. Significance of the normal distribution variables was estimated by the unpaired Student's $t$ test between two groups, and the non-normal distribution variables were analyzed by the Mann-Whitney U test. Kruskal-Wallis test and one-way analysis of variance served as non-parametric and parametric methods, respectively, to analyze differences among multiple groups. Correlation coefficient was calculated by Spearman's rank correlation coefficient. Two-sided Fisher exact test was performed to determine contingency tables. The $\mathrm{p}$ value was converted into FDR by Benjamini-Hochberg method. Survival curves of each subgroup in the dataset were plotted by Kaplan-Meier (KM) method. Logrank test was used to determine the statistical significance of the differences, which was defined as $p<0.05$. All the analyses were performed in $\mathrm{R}$ 3.4.3 using default parameters unless otherwise specified.

\section{Results}

\section{Identification of prognostic molecular subtypes} through comprehensive analysis of DNA methylation, CNVs and transcriptome

To identify the prognostic molecular subtypes of cervical cancer, genes showing PCGs, CNV and methylation with prognostic significance were screened based on univariate Cox proportional risk model, with a threshold of $\mathrm{p}<0.05$. Finally, 3897 genes, 4897 CNV regions and 20,144 CpG sites with significant prognostic association were obtained. Next, iClusterPlus was used for multi-omics cluster analysis, and identified three molecular subtypes, which were Cluster1 $(\mathrm{N}=77)$, Cluster2 $(\mathrm{N}=142)$ and Cluster3 $(\mathrm{N}=73)$. The results of multiomics clustering were compared with those obtained by separate hierarchical clustering (Fig. S1A), and the results showed that the three molecular subtypes determined by multi-omics clustering had some consistency with those obtained by single-omics clustering. For example, the methylation clustering results of Cluster1 and Cluster2 subgroups overlapped with those of multi-omics patients with Cluster1, suggesting that the molecular subtypes identified by multiple histologies combining the molecular characteristics of multiple histological data are richer in terms of information dimensions than those of individual histology, and that no one histology could reproduce the molecular subtypes identified by multiple histologies alone. The three subtypes had significant prognostic differences $(p=0.0047)$ (Fig. 1a), among them, Cluster1 showed the most favorable prognosis, while the Cluster3 had the worst prognosis. There was no significant difference in prognosis between the Cluster1 and Cluster2 groups (Fig. 1c), but the prognosis of Cluster3 was greatly worse than that of Cluster1 $(p=0.00247)$ and Cluster2 $(p=0.01297)$ (Fig. 1d, e). A total of 16 were obtained by the detection of the top 10 high-frequency mutated genes of each subtype (Fig. 1b). The distribution of these genes was similar in all three subtypes, suggesting a high degree of consistency in the genes associated with the most 


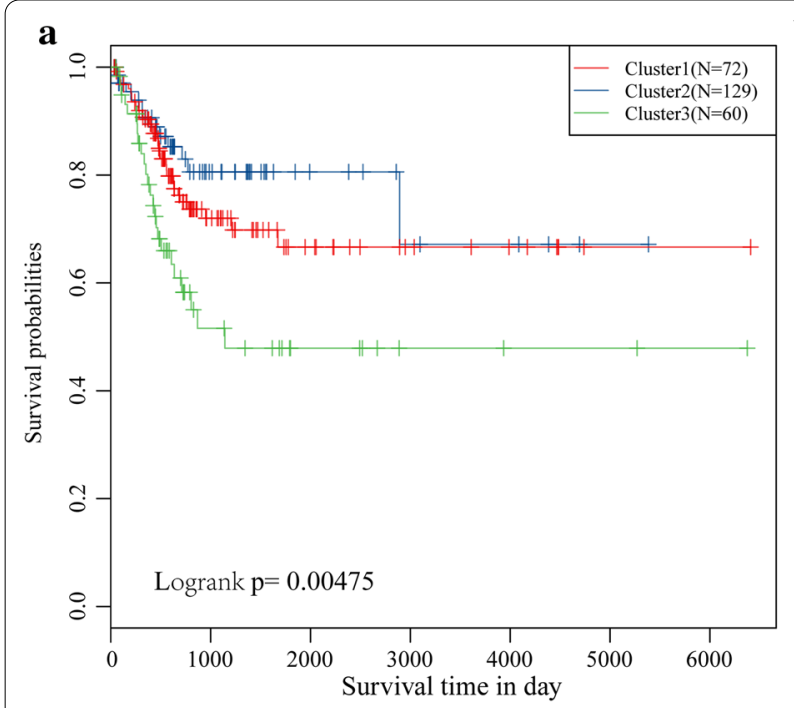

\section{b}
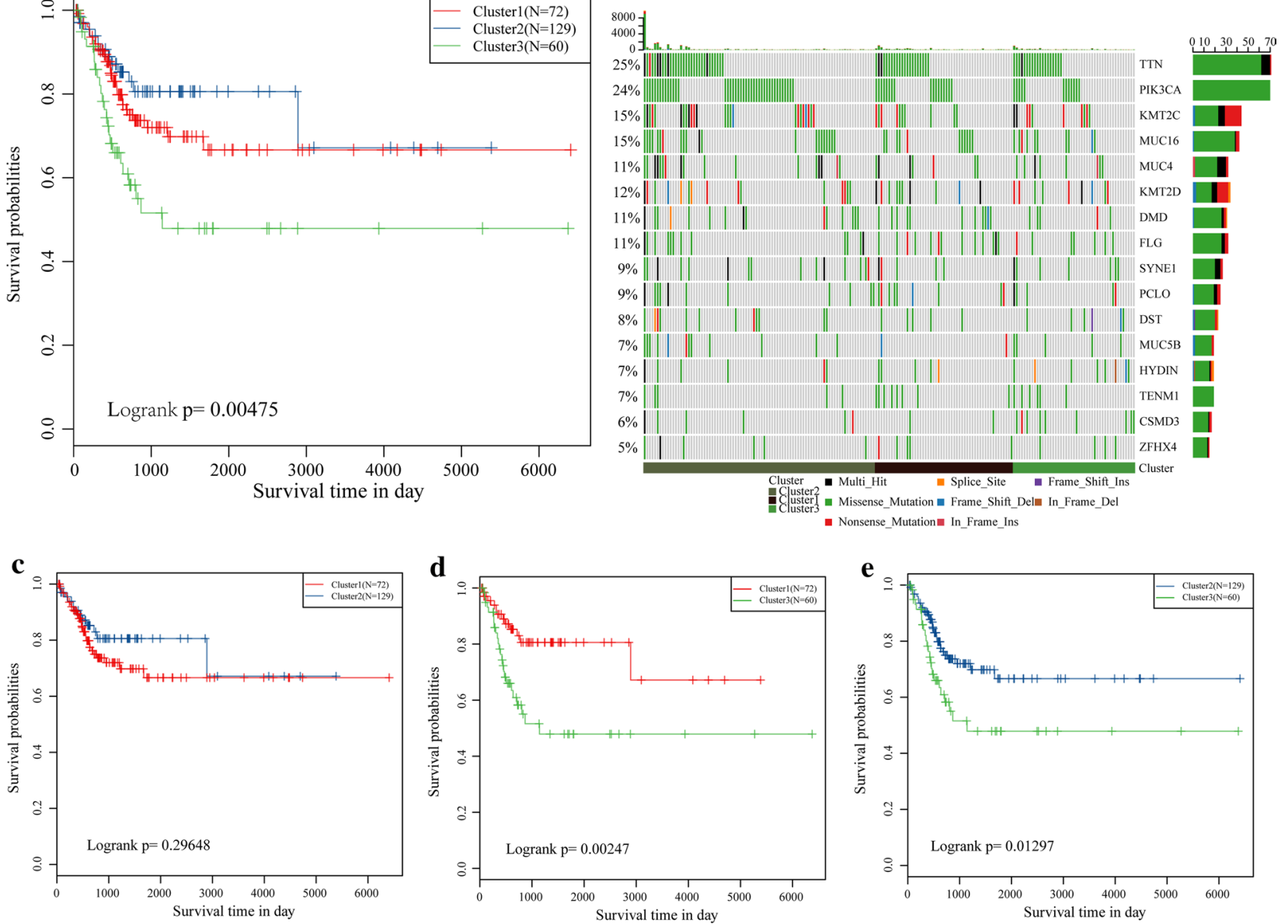

Fig. 1 Prognostic differences and molecular characteristics of the three molecular subtypes. a The total survival and prognosis of the three subtypes were different by KM curves. b The mutation distribution of high-frequency mutation genes in the three molecular subtypes of cervical cancer. The horizontal is molecular subtypes, ordinate (left) is the mutation frequency of the gene in each sample, ordinate (right) is mutation genes and colors in heat-map are different mutation types. c KM curve results showed there is no difference in prognosis between Cluster 1 and Cluster2. $d$ KM curve results showed there is significantly difference in prognosis between Cluster 1 and Cluster3. e KM curve results showed there is significant difference in prognosis between Cluster2 and Cluster3

common mutations in the three subtypes. In addition, TTN, PIK3CA, KMT2C, and MUC4 with higher mutation rates may suggest that these genes play a key role in canceration. The findings here indicated that subtype classification based on PCGs, methylation, and CNVs could predict the prognosis of patients with cervical cancer and have certain regulatory relationships at the levels of genome, epigenome, and transcription.

\section{Distribution of differentially expressed IncRNAs and PCGs in the three subtypes}

The differentially expressed lncRNAs and PCGs in three subtypes were determined. We found the small number of differentially expressed lncRNAs in both Cluster2 samples and Cluster3 samples. A total of 617 lncRNAs and 1395 PCGs were obtained from the three subtypes (Additional file 1: Table S1). The volcano map data of the differential expressions of the lncRNAs showed that in the three subtypes the number of up-regulated lncRNAs was generally smaller than those down-regulated (Fig. 2a-c). Moreover, the numbers of differentially expressed PCGs was much larger than that of lncRNAs (Fig. 2d). Finally, 584 lncRNAs closely related to cervical cancer were downloaded from LncRNADisease and Lnc2Cancer databases. We found that there was an obvious intersection between differentially expressed lncRNAs in the three subtypes and cervical cancer-related lncRNAs $(\mathrm{p}<0.0001)$ (Fig. 2g). Those data suggested that lncRNAs may play an important role in the heterogeneity and progression of cervical cancer. 

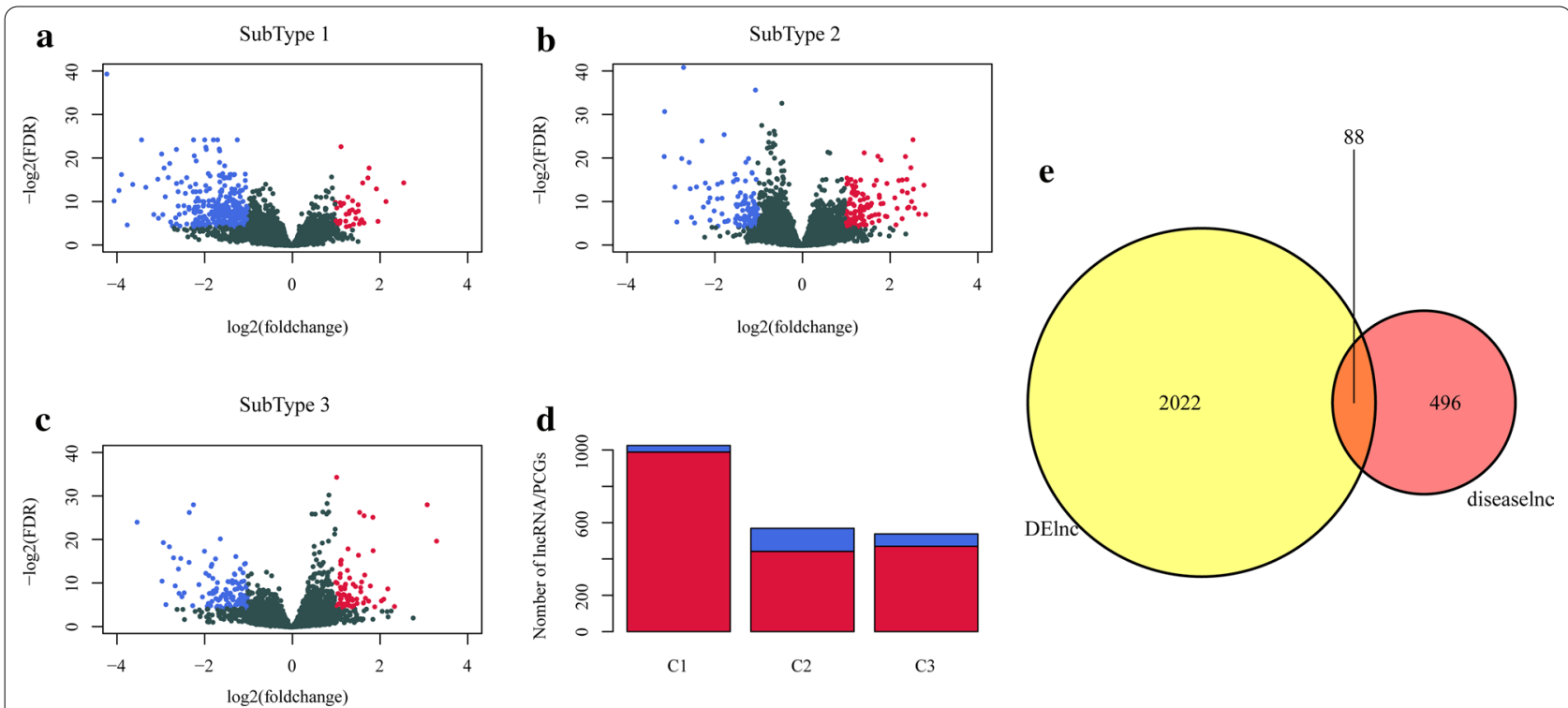

Fig. 2 Volcano gram and distribution of differentially expressed IncRNAs. a Volcano plot of differentially expressed IncRNAs in Cluster1. b Volcano plot of differentially expressed IncRNAs in Cluster2. c Volcano plot of differentially expressed IncRNAs in Cluster3. Red is up-regulated IncRNAs, blue is down-regulated IncRNAs. d The number of differentially expressed IncRNAs and PCGs genes in the three subtypes. Red is differentially expressed IncRNAs and blue is differentially expressed PCGs. e Venn diagram of the intersection of subtype-different IncRNAs and disease-related IncRNAs

\section{Identification of differentially co-expressed IncRNAs-PCGs and functional modules for IncRNAs enrichment in the three subtypes}

To identify differentially co-expressed lncRNA-PCGs, based on the differential expression profiles of lncRNAs and PCGs, outlier samples were removed by hierarchical clustering analysis. The linkage method was chosen as the complete method, and the clustering distance was calculated by Euclidean clustering, and those clustering distances exceeding five times the standard deviation $(80,572.36)$ were considered as outliers and were rejected. We finally obtained a total of 307 samples (Fig. 3a). WGCNA was conducted for building expression network, with $\beta=3$ (scale-free $\mathrm{R} 2=0.92$ ) of power as a Soft Thresholding to ensure a scale-free network (Fig. 3b, c). Here, a total of 26 modules were screened (Fig. 3d). The numbers of lncRNAs and PCGs in each module are shown in Additional file 2: Table S2. By analyzing the enrichment level of lncRNAs in each module, the number of lncRNAs and PCGs in each module was first counted, then the significance of lncRNA enrichment in each module was calculated using Fisher exact test with all DE-lncRNAs and DE-PCGs as background. The following four modules with significant enrichment of lncRNAs were identified: green, pink, magenta, and darkgreen (Fig. 3e). The KEGG pathway enrichment analysis of the PCGs in the four modules showed that the PCGs in these four modules were enriched to a total of 41 KEGG pathways (Fig. 4a). The pathways enriched by the four modules each showed limited intersection, and they tended to enrich to different pathways, suggesting that different modules may have different functions. The green module was found enriched to 19 KEGG pathways, and was mainly enriched to 553 signaling pathway, cAMP signaling pathway, Glucagon signaling pathway and some other pathways significantly related to tumorigenesis, development, tumor metabolism of cervical cancer (Fig. 4b). The pink module was enriched to the collecting duct acid secretion and SNARE interactions in vesicular transport pathway (Fig. 4c). As shown in Fig. 5D, the magenta module was enriched to 6 KEGG pathways, which are mainly the pathways related to cardiomyopathy such as Hypertrophic cardiomyopathy (HCM) and Dilated cardiomyopathy (DCM) (Fig. 4d). It is known that advanced cancers can easily induce great changes in metabolism, promote cardiac atrophy, and heart failure [37]. As shown in Fig. 5e, the darkgreen module was enriched to 7 KEGG pathways, mainly to PI3K-Akt signaling pathway, Nicotine addiction and other pathways related to tumorigenesis and development (Fig. 4e). These results indicated that lncRNAs may be directly or indirectly involved in important pathways of the tumorigenesis and development of cervical cancer.

\section{Abnormal expressions of IncRNAs were relevant to CNVs}

To analyze the relationship between lncRNA expressions and CNVs, IncRNAs copy number data were extracted from 292 cases of cervical cancer cases 


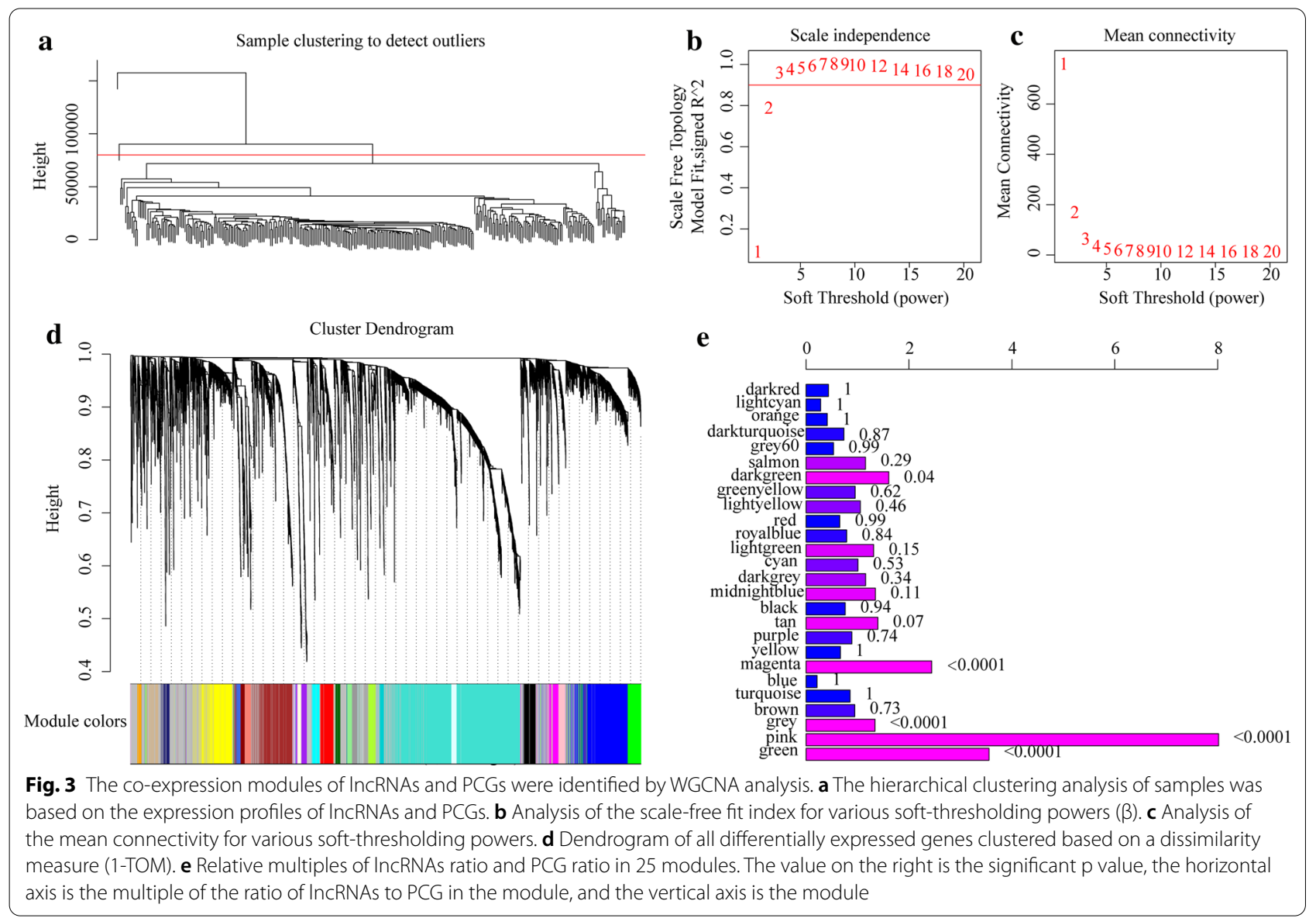

obtained from the TCGA, with copy number greater than 1 as the threshold of copy number amplification and less than -1 as the copy number deletion threshold. The ratio of copy number amplification and copy number deletion of each lncRNAs and its distribution in the genome were analyzed (Fig. 5a). Hwere, copy number deletion and copy number amplification showed different distributions on different chromosomes. For example, most copies of chromosome 3, 4, 8, 11 and 17 were absent, while some copies of chromosome 1 and 3 were increased. The association distribution between the expression profile of lncRNAs and copy number demonstrated an overall positive related trend, and the distribution in the actual situation was significantly larger than that in the random case $(\mathrm{p}<2.2 \mathrm{e}-16)$ (Fig. 5b). The frequently changing regions in the genome of cervical cancer patients were detected using GISTIC algorithm, and the data revealed many regions with significant copy number amplification or deletion of lncRNAs (Fig. 5c), suggesting that the abnormal copy number of lncRNAs may be related to the occurrence and development of cervical cancer. A total of 3 lncRNAs with a copy number ratio of more than $25 \%$ in each sample were identified, and their expression differences in copy number amplification/deletion and normal copied samples were analyzed. The data showed that the expressions of lncRNAs in the samples with copy amplification were significantly higher than that in the samples with normal copies (Fig. 5d), indicating that

\footnotetext{
(See figure on next page.)

Fig. 4 KEGG enrichment analysis of four IncRNA enrichment modules. a Network relationship of enrichment results of the four modules (green, pink, magenta and darkgreen). $\mathbf{b}$ Enrichment results of gene KEGG in green module. $\mathbf{c}$ KEGG enrichment results of genes in pink module. $\mathbf{d}$ KEGG enrichment results of genes in magenta module. e KEGG enrichment results of genes in darkgreen module. The color from red to blue represents the $p$ value from large to small, the size of the circle represents the number of genes in the enrichment pathway, with a larger circle representing more gene data
} 
$\mathbf{a}$
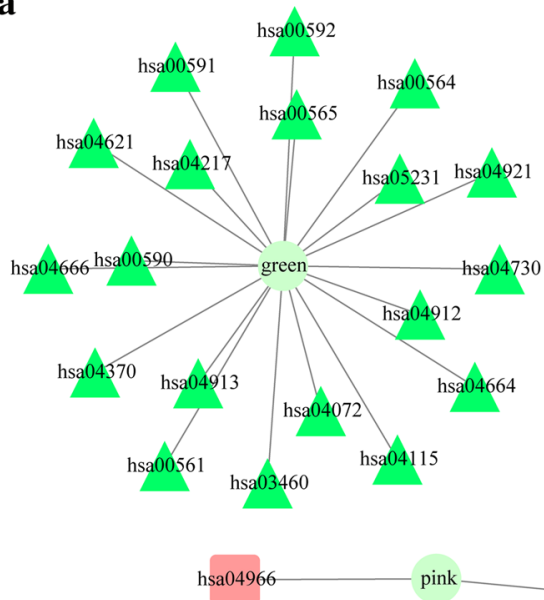
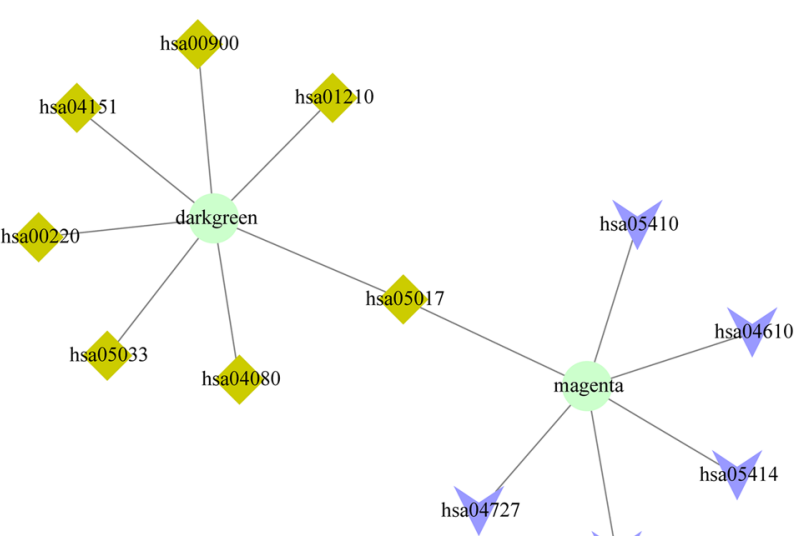

hsa 04260 b Necroptosis
Glycerophospholipid metabolism
Choline metabolism in cancer
Phospholipase D signaling pathway
Oxytocin signaling pathway
-like receptor signaling pathway
alpha-Linolenic acid metabolism
Linoleic acid metabolism
Ether lipid metabolism
Ovarian steroidogenesis
Fanconi anemia pathway
VEGF signaling pathway
Long-term depression
Glycerolipid metabolism
Arachidonic acid metabolism
Fc epsilon RI signaling pathway
p53 signaling pathway
GnRH signaling pathway

d

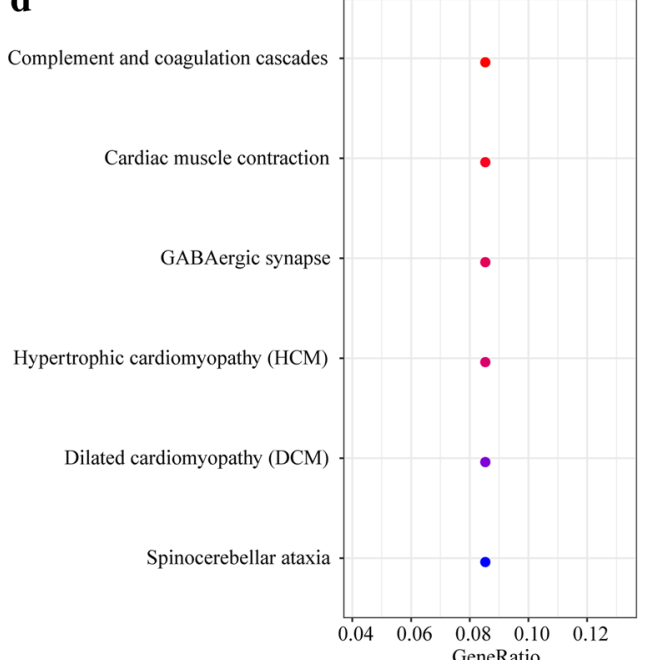

c

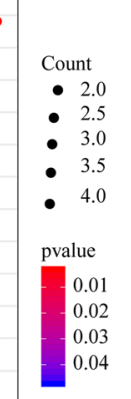

SNARE interactions in vesicular transport
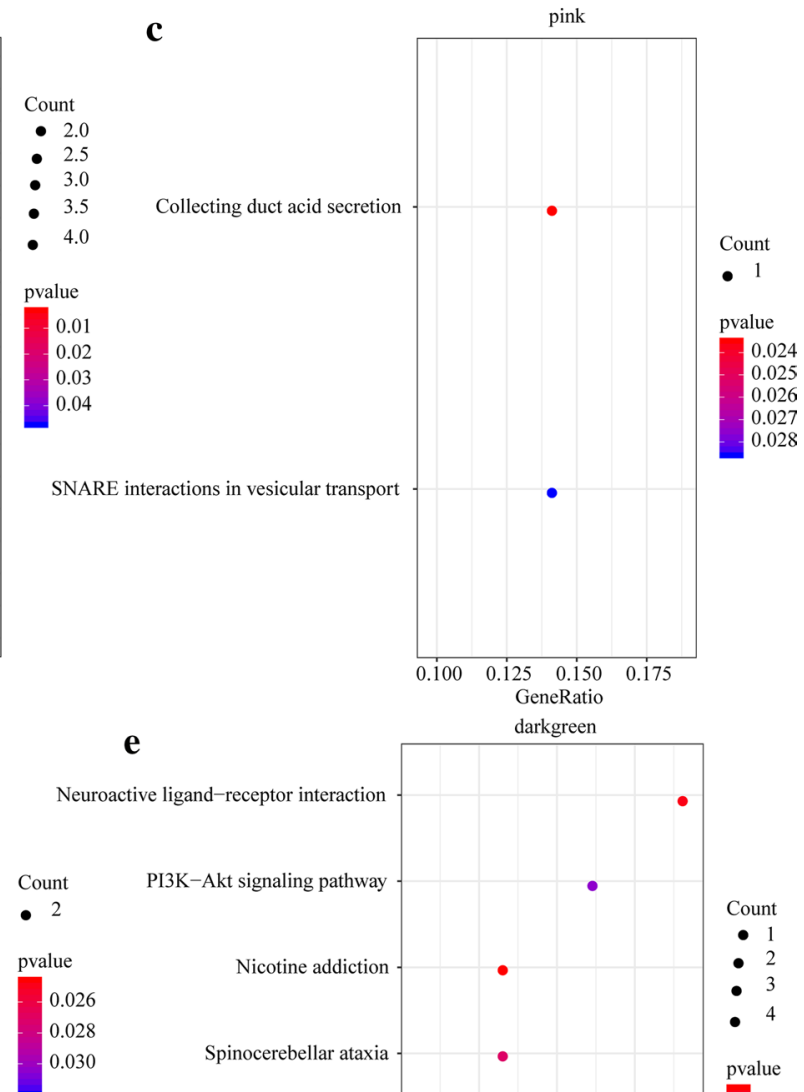

Collecting duct acid secretion

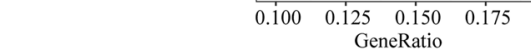

GeneRatio

darkgreen

active ligand-receptor interaction

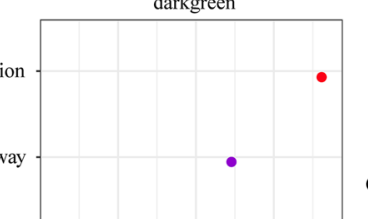

Count

- 1

- 2

- 3

pvalue

pvalue

0.03

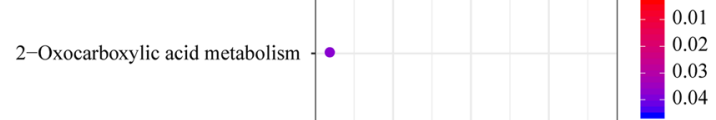

Terpenoid backbone biosynthesis 
the abnormal expressions of lncRNAs were related to the abnormal copy number.

\section{Identification of IncRNA prognostic markers with abnormal copy number in cervical cancer patients and establishment of a IncRNA signature}

A total of 575 lncRNAs with CNV greater than 15\% were selected. Univariate Cox analysis was performed to examine the relationship between these lncRNAs and OS, and we found that 41 lncRNAs significantly related to the cancer prognosis $p<0.01$ (Additional file 3: Table S3). LASSO Cox regression analysis was further performed to analyze the expression profiles of these lncRNAs. The change trajectory of each independent variable (Fig. 6a) demonstrated that the number of independent variable coefficients close to 0 gradually increased with the gradual increase of lambda (Fig. 6b). The model was built using tenfold cross-validation, and the confidence interval under each lambda was analyzed. When lambda $=0.0285$, we found that the model reached the highest performance, and there were 12 lncRNAs, which could serve as the potential prognostic markers. Furthermore, multivariate Cox survival analysis was performed, and 8 lncRNAs (Additional file 4: Table S4) with the lowest AIC value $(\mathrm{AIC}=546.05)$ were obtained as the final prognostic markers to establish a risk regression model:

RiskScore $=0.486 * \exp ^{\text {ENSG00000225855 }}+2.707 * \exp ^{\text {ENSG }}$ $00000273125+0.573 * \exp ^{\text {ENSG00000249306 }-2.601 * \exp ^{\text {ENSG0000 }}}$ $0253490+0.096 * \exp ^{\text {ENSG00000130600 }}+0.768 * \exp ^{\text {ENSG0000022 }}$ $9373-3.111 * \exp ^{\text {ENSG00000260898 }}+0.684 * \exp ^{\text {ENSG00000265096. }}$.

The relationship between risk score and lncRNA expressions was shown in Fig. 7c. As the risk score increased, the mortality rate of the samples also increased. 6 high-expressed lncRNAs associated with high risk score were a risk factors, while 2 lowexpressed lncRNAs associated with high risk score were protective factors (Fig. 6c). One-year, three-year and five-year of ROC analysis and prediction showed that the model had a high AUC area, and they all had an AUC above 0.75 (Fig. 6d). Finally, Zscore of the risk score was calculated to divide the samples with a risk score higher than zero into high-risk group $(\mathrm{N}=114)$ and with a score lower than zero into low-risk groups
$(\mathrm{N}=121)$. Interestingly, the samples in the high-risk group showed significantly worse prognosis than those in the low-risk group $(\mathrm{p}<0.0001, \mathrm{HR}=3.406,95 \% \mathrm{CI}$ : 1.912-6.064) (Fig. 6e).

In addition, $50 \%$ of the 292 samples were randomly selected and repeated one thousand times. The model was applied to the prognostic prediction of these one thousand samples, and the prognostic significance p-values were calculated for each calculation (Additional file 5: Fig. S1B). The prognostic predictive power in these samples was observed to show significant differences in the prognostic significance p-values for each of the one thousand random samples. In addition, we also analyzed the correlation between 8 lncRNAs (Additional file 5: Fig. S1C), and only a few were significantly correlated, as expected there was no covariance among them.

\section{Prognostic model validation and functional analysis of the 8-IncRNA model}

To verify the performance of the $8 \mathrm{CNV}$-related lncRNAs in predicting the prognosis of cervical cancer, the risk scores of each sample in all TCGA data sets were calculated according to the expressions of the samples, and the predictive classification efficiency of 1-year, 3-year, and 5-year AUC was determined. The results showed that the model had a high AUC line area above 0.75 (Additional file 6: Fig. S2a). The samples were divided into high- and low-risk groups according to the threshold, and we found that the prognosis of the high-risk group was significantly worse than that of the lowrisk group $(\mathrm{p}<0.0001, \mathrm{HR}=3.133,95 \% \mathrm{CI}: 1.854-5.291)$ (Additional file 6: Fig. S2a). To further verify the robustness of the model, the GSE44001 data of GPL14951 platform was downloaded. The average AUC of 1-year, 3-year, and 5-year ROC was all higher than 0.6 (Additional file 6: Fig. S2c). Moreover, we also assessed the prognosis of the samples in high-risk group and low-risk group, and observed that the prognosis of the high-risk group was significantly worse than that of the low-risk group $(\mathrm{p}=0.036, \mathrm{HR}=1.957$, 95\%CI: 1.032-3.707) (Additional file 6: Fig. S2d). These data indicated that the performance model of 8-IncRNA signature model had a great robustness. To investigate the relationship between Riskscore and biological functions of

\footnotetext{
(See figure on next page.)

Fig. 5 Abnormal expressions of IncRNAs were positively correlated with abnormal copy numbers. a Distribution of IncRNA copy number amplification and deletion in genome. $\mathbf{b}$ The correlation distribution between IncRNA expressions and CNVs, light blue represents the distribution under random conditions, orange represents the distribution under actual conditions, t-test was used to examine the difference. c The IncRNAs located in the focal CNA peaks are cervical cancer-related. False-discovery rates and scores from GISTIC 2.0 for alterations (x-axis) are plotted against genome positions (y-axis); dotted lines indicate the centromeres. The deletions (right, blue) and amplifications (left, red) of IncRNAs genes are also shown. The green line represents 0.05 (FDR) as cut-off point that determines significance. $\mathbf{d}$ The expressions of IncRNAs in the samples with copy amplification are significantly higher than that in the samples with normal copies
} 
a

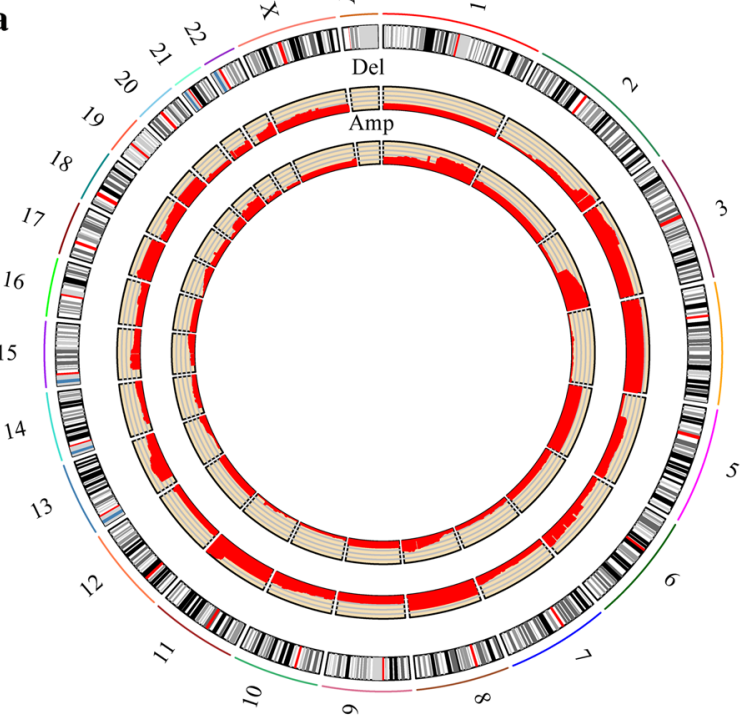

c

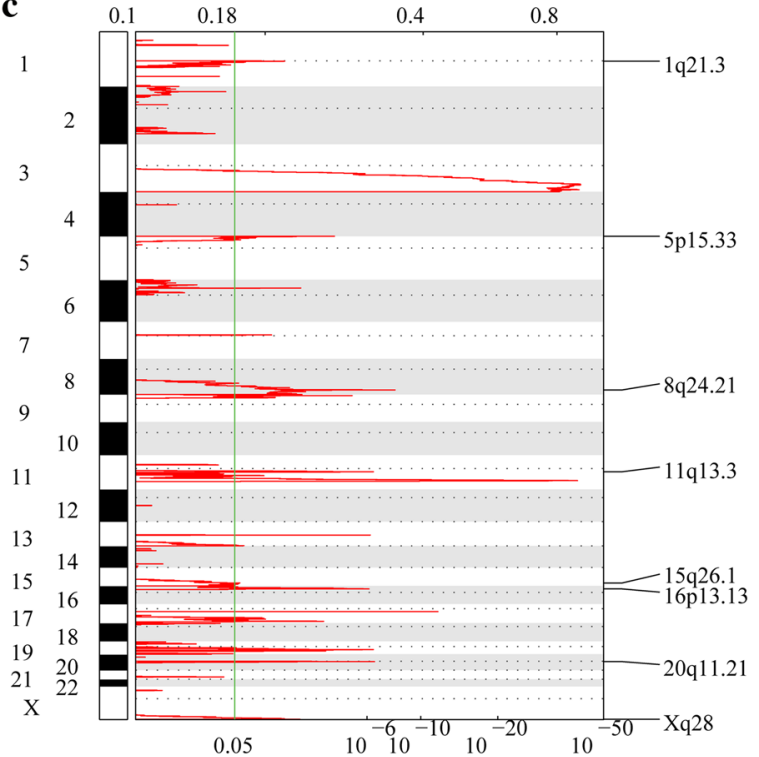

b
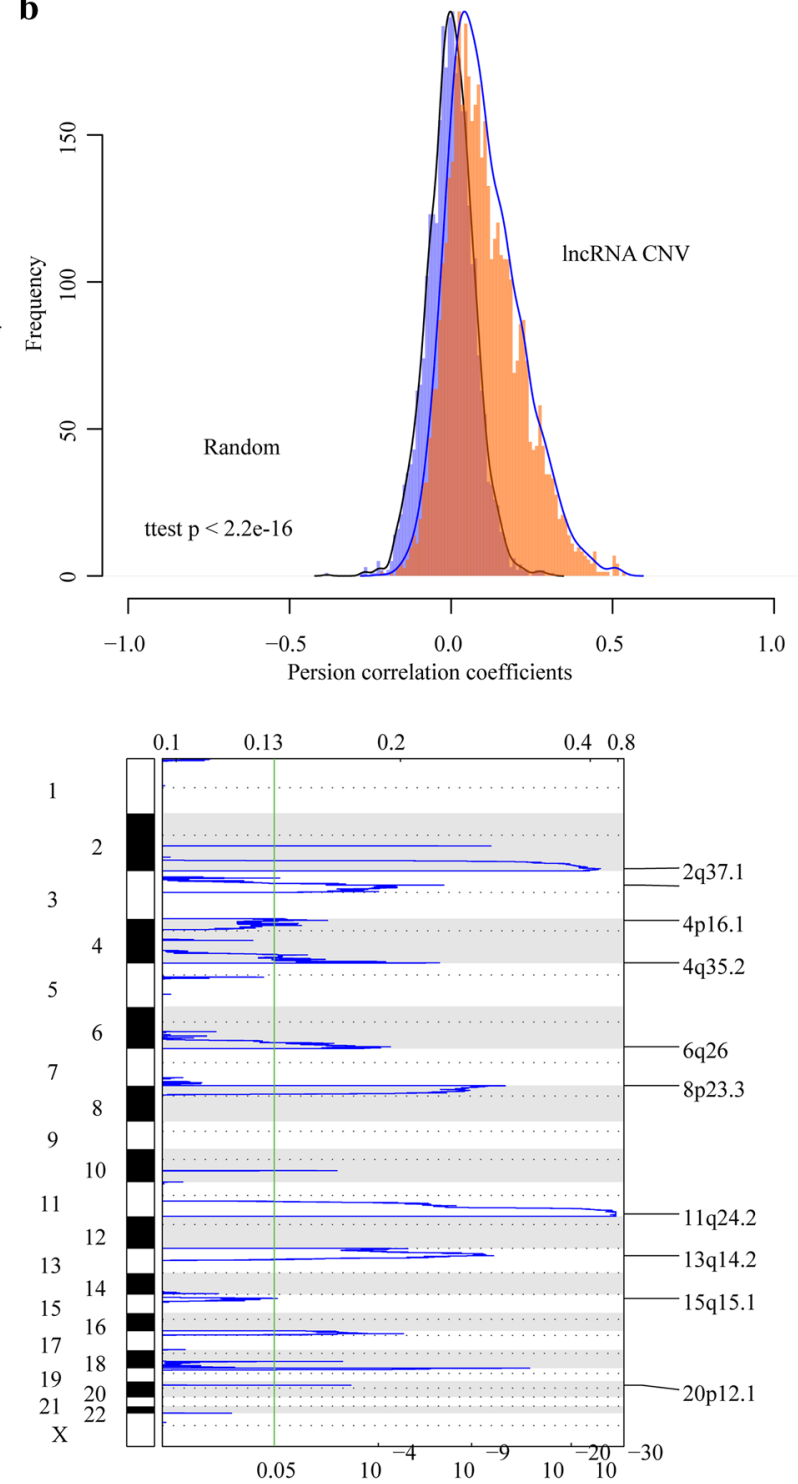

d Kruskal- Wallis test $\mathrm{p}=8.2 \mathrm{e}-07$
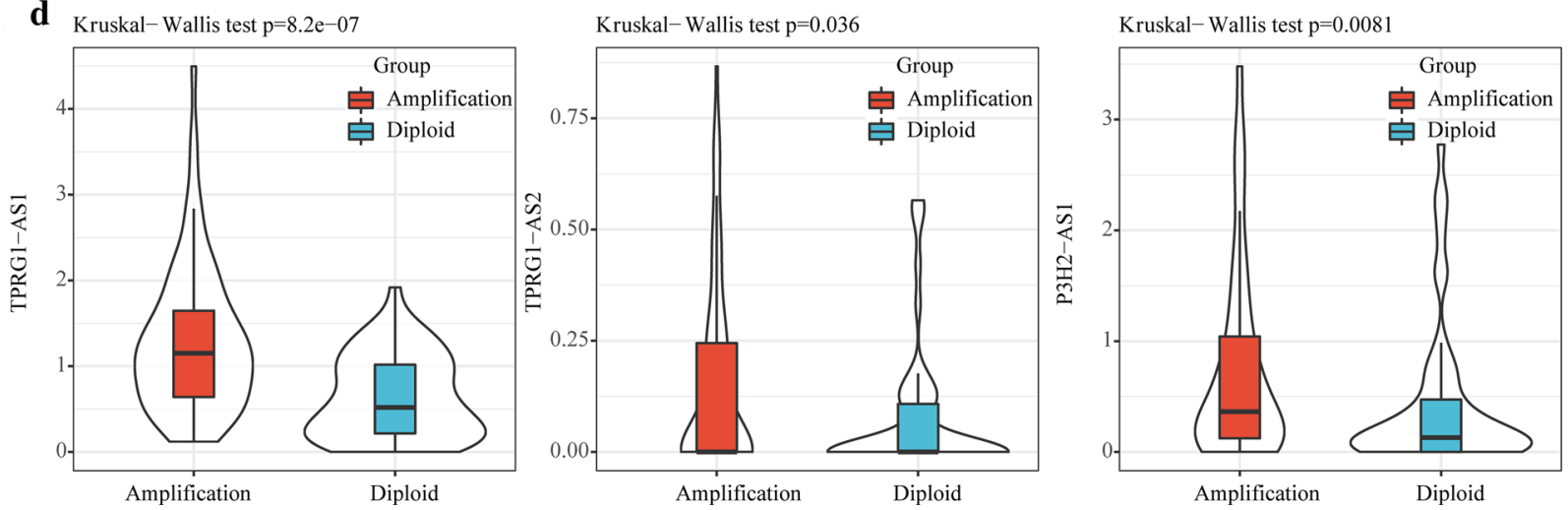

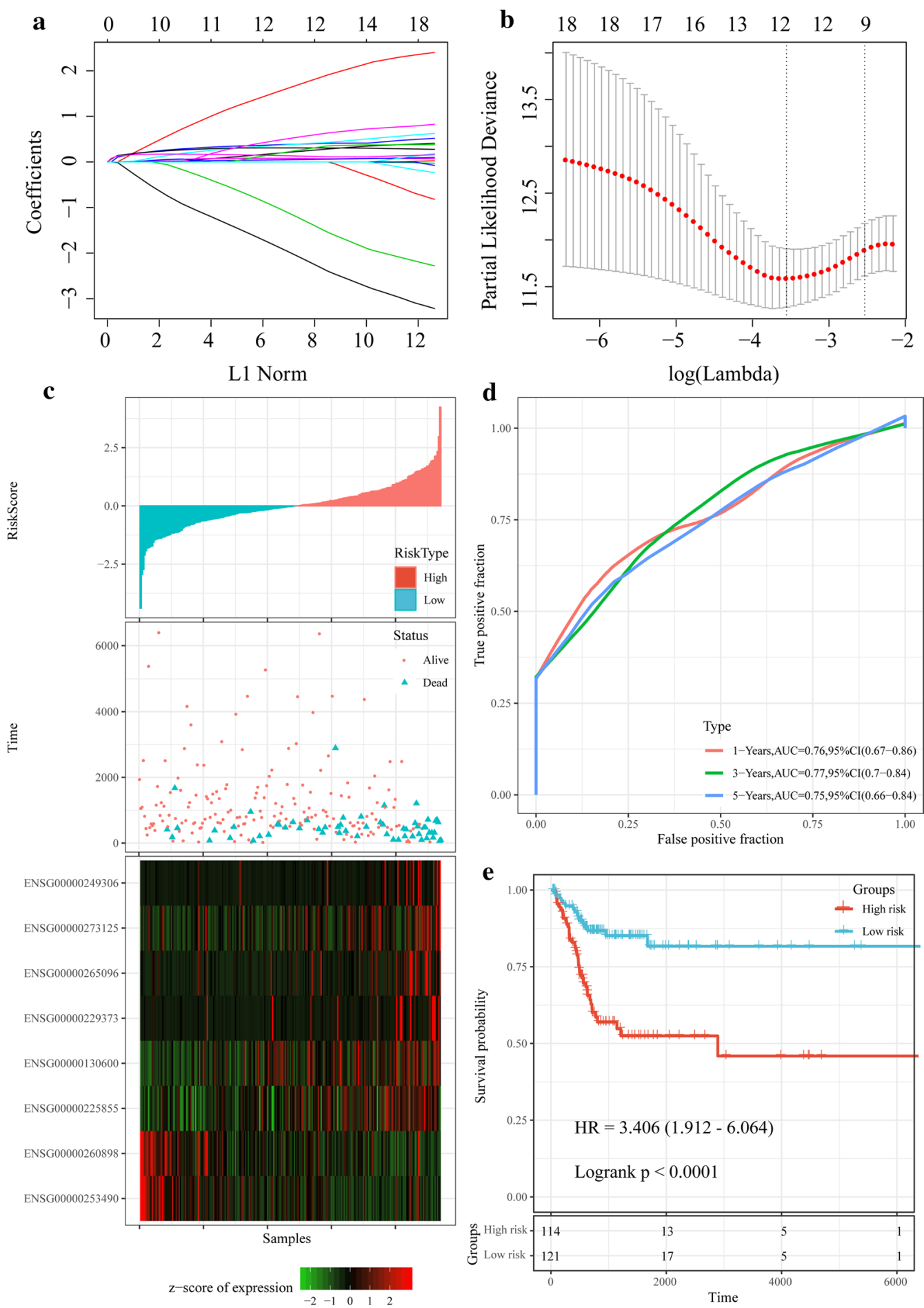

Fig. 6 Screening of IncRNA prognostic markers and establishment of prognostic models. a The number of genes is increasing as lambda increases, the horizontal axis represents the log value of the independent variable lambda, and the vertical axis represents the coefficient of the independent variable. $\mathbf{b}$ Confidence interval under each lambda. c Risk score, survival time, survival status and expression of the 8-IncRNA signature in the training set. $\mathbf{d}$ ROC curve and AUC of the 8-InCRNA signature in training set. e KM survival curve distribution of the 8-IncRNA signature in the training set 
different samples, risk score was correlated with the enrichment score of KEGG pathways in each sample. KEGG pathways with a correlation greater than 0.4 and $\mathrm{FDR}<0.01$ contained 20 pathways (Additional file 6: Fig. S2e). Interestingly, these 20 pathways were negatively correlated with Riskscore and mainly included T CELL RECEPTOR SIGNALING PATHWAY, B_CELL_RECEPTOR_SIGNALING_ PATHWAY, CYTOSOLIC_DNA_SENSING_PATHWAY, CYTOKINE_CYTOKINE_RECEPTOR_INTERACTION and some other immune-related pathways. The results suggested that the samples from the high-risk group and the low-risk group may have different immune microenvironments, and that these lncRNAs may be involved in tumor progression by affecting immune-related pathways.

\section{Comparison of 8-IncRNA prognosis model with clinical features and the existing models}

To identify the independence of the 8-lncRNA signature, the relationship between $\mathrm{T}, \mathrm{N}, \mathrm{M}$ stage, age, and risk score and prognosis was analyzed by Univariate and multivariate Cox regression analysis. Univariate Cox regression analysis showed that $\mathrm{N}$ stage, TNM stage and risk score were significantly related to survival (Additional file 7: Fig. S3a), However, multivariate Cox regression analysis found that only risk score $(\mathrm{p}<0.0001$, $\mathrm{HR}=3.425$, 95\% CI: 1.922-6.104) was significantly correlated with prognosis (Fig. S3B). Thus, the data revealed that the 8-lncRNAs signature can serve as a prognostic predictor independent of clinical characteristics. Furthermore, we compared the 8-lncRNA signature with four recently reported prognostic-related risk models, namely, the 4-lncRNA signature by sun et al. [33], the 10-IncRNA signature by Shen et al. [34], the 9-lncRNA signature by $\mathrm{Yu}$ et al. [35], and the 6-lncRNA signature by Luo et al. [36]. In order to ensure the comparability of the models, according to the corresponding genes in these 4 models, the same method was used to calculate the risk score of each cervical cancer sample in the TCGA dataset and ROC of each model, and KM survival curve was plotted. Although the prognosis of the Risk-H and Risk-L group samples of the four models were significantly different, the AUC prediction accuracy of the four models was lower than that of our 8-lncRNA model (Additional file 7: Fig. S3C-F). The restricted mean survival of these four models was also compared with our 8-lncRNA model. We observed that the 8-lncRNA model was more accurate in predicting a longer follow-up time and the $\mathrm{C}$-index was higher than the other four models (Additional file 7: Fig. S3g). Similarly, the DCA results showed that the risk score of the 8-lncRNA model developed in this study was far more indicative than the other four subtypes (Additional file 7: Fig. $\mathrm{S} 3 \mathrm{H}$ ). These results suggested that the 8-lncRNA signature is a new reliable prognostic marker independent of clinical stages.

\section{Discussion}

With the rapid development of next-generation sequencing and mass spectrometry technology, the biological complexity of tumors and the genetic etiology of cervical cancer has been increasingly elucidated and developed. In this study, we identified three prognostic molecular subtypes of cervical cancer based on multidimensional omics analysis, and screened subtype-specific lncRNAs and PCGs. Based on weighted co-expression analysis of the type-specific lncRNAs, three subtypes were found significantly related to the metabolism, immunity and other pathways of cervical cancer, suggesting that the subtype-specific lncRNAs may play different roles in the occurrence and development of cervical cancer and have different effects on the tumor progression. The relationship between the expressions of these lncRNAs and copy numbers were systematically analyzed, and the results demonstrated that the expressions of these lncRNAs were highly correlated with copy number amplification and deletion. LncRNAs with copy amplification tended be high-expressed, while those with deletion tended to be low-expressed. Based on CNVs and lncRNA expressions, 8 lncRNAs were identified as the potential prognostic markers for cervical cancer. The 8-lncRNA signature showed an accurate predictive performance in both the training set and the verification set, and can therefore be used as an independent prognostic factor for cervical cancer. Compared with other existing lncRNA signatures, our 8-lncRNA signature showed more stable predictive performance and higher AUC.

Past studies have shown that integrating multiomics clustering, such as iCluster, intNMF, Similar Network Fusion (SNF), could reveal tumor heterogeneity and actual prognostic features. In this study, multidimensional data processing was performed using iCluster based on a joint latent variable model of cervical cancer. The most noticeable feature of iCluster is the combination of estimated unobserved variables such as copy number data, mRNA expression data, and methylation, also iCluster can reduce the dimensionality of the data set without changing the sample size. Our algorithm matrix had a cohort of 292 cervical cancer patients, and the genome and epigenome contained three omics data, including mRNA, CNV, and methylation, which showed unique molecular characteristics and prognostic relevance. The prognosis of the Cluster3 subtype was poor, while the results of the Cluster2 subtype were the most favorable. Recent studies found that CNVcor and methylation-related genes (METcor) are significantly co-regulated, moreover, the integration of CNVcor and 
METcor genes have identified three molecular subtypes in liver cancer [38]. This suggested the significance of establishing a comprehensive prognostic molecular subtype based on genome and epigenome. In this study, we compared mutation profiles of the three molecular subtypes, and discovered that TTN, PIK3CA, KMT2C and MUC4 mutations were more common than other genes. Noticeably, the PIK3CA mutation is related to the resistance of cervical cancer to the treatment [39]. MUC4, which is a transmembrane glycoprotein expressed higher in cervical dysplasia than in benign cervical epithelium, is also related to lymph node metastasis of cervical cancer [40].

The occurrence and development of cancerous changes are often associated with enormous genomic mutations, including small size mutations (SNPs) and CNVs, loss of copy number, duplication, and amplification. CNV is a hallmark of cancer and often causes abnormal copy numbers, including amplification, increase, loss, and deletion. CNV plays an important role in regulating the expressions of PCGs and lncRNAs and the activation of multiple signaling pathways. It is known that CNV has critical functions in the development of various tumors, such as ovarian cancer [41], breast cancer [42], endometrial cancer [42].

Although we identified potential lncRNAs predictive of the prognosis of cervical cancer from large samples by applying bioinformatics techniques, some limitations still exist in this study. Firstly, the sample lacked clinical follow-up information, thus, factors such as the presence of other health conditions were not considered during the identification of the biomarkers. Secondly, the results obtained by bioinformatics analysis alone were not convincing enough, which requires further experimental verification. Therefore, genetic and experimental studies with larger sample sizes and experimental validation are needed.

\section{Conclusion}

To conclude, we identified prognostic-associated molecular subtypes by conducting Multi-omics analysis. 8 lncRNAs with abnormal copy numbers were determined as prognostic markers, and an 8-lncRNA prognostic layering system was developed. The 8-IncRNA signature showed a high AUC in both training and validation sets, and was independent of clinical features. Compared with clinical features, the 8-lncRNA classifier could greatly improve the accuracy of predicting survival risk. Therefore, this classifier can be used as a reliable molecular diagnostic model in evaluating the prognostic risk of patients with cervical cancer.

\section{Abbreviations}

CNV: Copy number variation; IncRNAs: Long non-coding RNAs; mRNAs: Messenger RNAs; PCGs: Protein-coding genes; LASSO: Least absolute shrinkage and selection operator (); WES: Whole exome sequencing; TCGA: The Cancer Genome Atlas; GEO: Gene Expression Omnibus; GSEA: Gene Set Enrichment Analysis; WGCNA: Weighted gene co-expression network analysis; DE: Differential expression; TOM: Topological overlap matrix; KEGG: Kyoto Encyclopedia of Genes and Genomes; SCNA: Somatic cell copy number change; OS: Overall survival; AIC: Akaike information criterion; ROC: Receiver operating characteristic; C-index: Concordance index; DCA: Decision curve analysis; KM: KaplanMeier; HCM: Hypertrophic cardiomyopathy; DCM: Dilated cardiomyopathy.

\section{Supplementary Information}

The online version contains supplementary material available at https://doi. org/10.1186/s12967-021-02705-9.

Additional file 1: Table S1. Distribution of differentially expressed IncRNA and PCGs in three subtypes.

Additional file 2: Table S2. CGPs and IncRNA in 26 modules

Additional file 3: Table S3. Prognostic information of 41 Incrnas with significant prognosis.

Additional file 4: Table S4. Information of 8-IncRNA signature.

Additional file 5: Figure S1. Advantage of multi-omics. a The results of multi-omics clustering were compared with those obtained by separate hierarchical clustering. b: Model was applied to the prognostic prediction of these one thousand samples, and the prognostic significance $p$-values were calculated for each calculation. c The correlation between 8 IncRNAs were analyzed.

Additional file 6: Figure S2. Prognostic model validation and functional analysis of the 8-IncRNA model. a ROC curve of the 8-IncRNA model in all TCGA datasets. Abscissa means false positive fraction, ordinate means true positive fraction. $\mathbf{b}$ KM survival curve distribution of the 8-IncRNA model in the high- and low-risk groups in all TCGA datasets. Abscissa means time, ordinate means survival probability. c ROC curve of the 8-IncRNA model in GSE44001 dataset. Abscissa means false positive fraction, ordinate means true positive fraction. $\mathbf{d}$ KM survival curve distribution of the 8-IncRNAs in the high- and low- risk group in GSE44001 datasets. Abscissa means time, ordinate means survival probability. e KEGG Pathway was the most correlated with the 8 -IncRNA model, and the circle size in the figure indicates the correlation.

Additional file 7: Figure S3. Comparison of the 8-IncRNA prognosis model with clinical features and the existing models a Forest characteristics of clinical features and risk score using univariate survival analysis. b Forest characteristics of clinical characteristics and risk score using multivariate survival analysis, and among them, orange-red represents a significant prognostic correlation. c ROC curve and KM curve of a 4-IncRNA signature in TCGA dataset. $\mathbf{d}$ ROC curve and KM curve of a 10-IncRNA signature in TCGA dataset. e ROC curve and KM curve of a 9-InCRNA signature in TCGA dataset. $\mathbf{f} O C$ curve and KM curve of a 6-IncRNA signature in TCGA dataset. Left: Abscissa means false positive fraction, ordinate means true positive fraction. Right: Abscissa means time, ordinate means survival probality. G: Comparison of restricted mean survival of five prognostic risk models. Abscissa means restricted mean survival, ordinate means percentiles of marker. $\mathbf{h}$ Comparison of decision curve analysis of the five prognostic risk models. Abscissa means threshold probability, ordinate means net benefit.

\section{Acknowledgments}

None.

\section{Authors' contributions}

Conception and design of the research: JH, JQ; Acquisition of data: YF, HO; Analysis and interpretation of data: QZ, YC; Statistical analysis: ZW; Obtaining funding: $\mathrm{JH}, \mathrm{HO}$; Drafting the manuscript: $M L, W Y$; Revision of manuscript for 
important intellectual content: JH. All authors read and approved the final manuscript.

\section{Funding}

This work was supported by the National Key Research and Development Program of China (2018YFC1003702 and 2018YFC1004400), the Beijing Nova Program (Z201100006820010), the National Natural Science Funds of China (82071658 and 31800624), Key Clinical Program of Peking University Third Hospital (BYSY2017031), State Key Laboratory of Molecular Developmental Biology (2020-MDB-KF-18), Beijing Natural Science Foundation (7204327) and Capital's Funds for Health Improvement and Research (2020-4-40916).

\section{Availability of data and materials}

The data that support the findings of this study are available from the corresponding author upon reasonable request.

\section{Ethics approval and consent to participate}

Not applicable.

\section{Consent for publication}

Not applicable.

\section{Competing interests}

The authors declare that they have no competing interests.

\section{Author details}

${ }^{1}$ Institute of Systems Biomedicine, School of Basic Medical Sciences, Peking University Health Science Center, Peking University, HaiDian District, No. 38 XueYuan Road, Beijing 100191, China. ${ }^{2}$ Center for Reproductive Medicine, Department of Obstetrics and Gynecology, Peking University Third Hospital, HaiDian District, No. 49 North HuaYuan Road, Beijing 100191, China. ${ }^{3}$ National Clinical Research Center for Obstetrics and Gynecology, Beijing 100191, China. ${ }^{4}$ Key Laboratory of Assisted Reproduction, Ministry of Education, Beijing 100191, China. ${ }^{5}$ Beijing Key Laboratory of Reproductive Endocrinology and Assisted Reproductive Technology, Beijing 100191, China. ${ }^{6}$ Department of Orthopedics, Peking University Third Hospital, Beijing 100091, China. ${ }^{7}$ Beijing Key Laboratory of Spinal Disease Research, Beijing 100191, China. ${ }^{8}$ Key Laboratory for Major Obstetric Diseases of Guangdong Province, The Third Affiliated Hospital of Guangzhou Medical University, Guangzhou 510150, China. ${ }^{9}$ State Key Laboratory of Molecular Developmental Biology, Institute of Genetics and Developmental Biology, Chinese Academy of Sciences, Beijing, China.

\section{Received: 18 October 2020 Accepted: 12 January 2021}

Published online: 08 February 2021

\section{References}

1. Cancer Genome Atlas Research N, Albert Einstein College of M, Analytical Biological S, Barretos Cancer H, Baylor College of M, Beckman Research Institute of $\mathrm{City}$ of $\mathrm{H}$, et al. Integrated genomic and molecular characterization of cervical cancer. Nature. 2017;543(7645):378-84.

2. Kent A. HPV Vaccination and Testing. Rev Obstet Gynecol. 2010;3(1):33-4.

3. Murillo R, Herrero R, Sierra MS, Forman D. Cervical cancer in Central and South America: Burden of disease and status of disease control. Cancer Epidemiol. 2016:44(Suppl 1):S121-30.

4. Bray F, Ferlay J, Soerjomataram I, Siegel RL, Torre LA, Jemal A. Global cancer statistics 2018: GLOBOCAN estimates of incidence and mortality worldwide for 36 cancers in 185 countries. CA Cancer J Clin. 2018;68(6):394-424.

5. Wang SS, Hildesheim A. Chapter 5: Viral and host factors in human papillomavirus persistence and progression. J Natl Cancer Inst Monogr. 2003(31):35-40

6. Li X. Emerging role of mutations in epigenetic regulators including MLL2 derived from The Cancer Genome Atlas for cervical cancer. BMC Cancer. 2017;17(1):252.

7. Nakamura Y. DNA variations in human and medical genetics: 25 years of my experience. J Hum Genet. 2009;54(1):1-8.
8. Conrad DF, Pinto D, Redon R, Feuk L, Gokcumen O, Zhang Y, et al. Origins and functional impact of copy number variation in the human genome. Nature. 2010;464(7289):704-12.

9. Loharamtaweethong K, Supakatitham C, Vinyuvat S, Puripat N, Tanvanich S, Sitthivilai U. Prognostic significance of PD-L1 protein expression and copy number gains in locally advanced cervical cancer. Asian Pac J Allergy Immunol. 2019.

10. Joharinia N, Farhadi A, Hosseini SY, Safaei A, Sarvari J. Association of HPV16 and 18 genomic copies with histological grades of cervical lesions. Virusdisease. 2019;30(3):387-93.

11. Gonzalez E, Kulkarni H, Bolivar H, Mangano A, Sanchez R, Catano G, et al. The influence of CCL3L1 gene-containing segmental duplications on HIV-1/AIDS susceptibility. Science. 2005;307(5714):1434-40.

12. Aitman TJ, Dong R, Vyse TJ, Norsworthy PJ, Johnson MD, Smith J, et al. Copy number polymorphism in Fcgr3 predisposes to glomerulonephritis in rats and humans. Nature. 2006;439(7078):851-5.

13. Spizzo R, Almeida MI, Colombatti A, Calin GA. Long non-coding RNAs and cancer: a new frontier of translational research? Oncogene. 2012;31(43):4577-87.

14. Mitra SA, Mitra AP, Triche TJ. A central role for long non-coding RNA in cancer. Front Genet. 2012;3:17.

15. Fatica A, Bozzoni I. Long non-coding RNAs: new players in cell differentiation and development. Nat Rev Genet. 2014;15(1):7-21.

16. Zhang Y, Wu D, Wang D. Long non-coding RNA ARAP1-AS1 promotes tumorigenesis and metastasis through facilitating proto-oncogene c-Myc translation via dissociating PSF/PTB dimer in cervical cancer. Cancer Med. 2020.

17. Wang J, Zhu Z, Qiu H, Liu C, Chang X, Qi Y, et al. LncRNA NKILA inhibits the proliferation and promotes the apoptosis of CSCC cells by downregulating miRNA-21. J Cell Physiol. 2020.

18. Gao F, Feng J, Yao H, Li Y, Xi J, Yang J. LncRNA SBF2-AS1 promotes the progression of cervical cancer by regulating miR-361-5p/FOXM1 axis. Artif Cells Nanomed Biotechnol. 2019;47(1):776-82.

19. Gibb EA, Vucic EA, Enfield KS, Stewart GL, Lonergan KM, Kennett JY, et al. Human cancer long non-coding RNA transcriptomes. PLoS ONE. 2011;6(10):e25915.

20. Sun YV, Hu YJ. Integrative Analysis of Multi-omics Data for Discovery and Functional Studies of Complex Human Diseases. Adv Genet. 2016:93:147-90.

21. The TCGA Legacy. Cell. 2018;173(2):281-2.

22. Harrow J, Frankish A, Gonzalez JM, Tapanari E, Diekhans M, Kokocinski F, et al. GENCODE: the reference human genome annotation for The ENCODE Project. Genome Res. 2012;22(9):1760-74.

23. Chen YA, Lemire M, Choufani S, Butcher DT, Grafodatskaya D, Zanke BW, et al. Discovery of cross-reactive probes and polymorphic CpGs in the Illumina Infinium HumanMethylation450 microarray. Epigenetics. 2013;8(2):203-9.

24. Shi Q, Zhang C, Peng M, Yu X, Zeng T, Liu J, et al. Pattern fusion analysis by adaptive alignment of multiple heterogeneous omics data. Bioinformatics. 2017;33(17):2706-14.

25. Pierre-Jean M, Deleuze JF, Le Floch E, Mauger F. Clustering and variable selection evaluation of 13 unsupervised methods for multi-omics data integration. Brief Bioinform. 2020;21(6):2011-30.

26. Li Z, Jiang C, Yuan Y. TCGA based integrated genomic analyses of ceRNA network and novel subtypes revealing potential biomarkers for the prognosis and target therapy of tongue squamous cell carcinoma. PLOS ONE. 2019;14(5):e0216834.

27. Bao Z, Yang Z, Huang Z, Zhou Y, Cui Q, Dong D. LncRNADisease 2.0: an updated database of long non-coding RNA-associated diseases. Nucleic acids research. 2019;47(D1):D1034-D7.

28. Gao Y, Wang P, Wang Y, Ma X, Zhi H, Zhou D, et al. Lnc2Cancer V2.0: updated database of experimentally supported long non-coding RNAs in human cancers. Nucleic acids research. 2019;47(D1):D1028-D33.

29. Langfelder P, Horvath S. WGCNA: an R package for weighted correlation network analysis. BMC Bioinformatics. 2008;9:559.

30. Mermel CH, Schumacher SE, Hill B, Meyerson ML, Beroukhim R, Getz G. GISTIC2.0 facilitates sensitive and confident localization of the targets of focal somatic copy-number alteration in human cancers. Genome biology. 2011;12(4):R41.

31. Simon N, Friedman J, Hastie T, Tibshirani R. Regularization Paths for Cox's Proportional Hazards Model via Coordinate Descent. J Stat Softw. 2011;39(5):1-13. 
32. Hanzelmann S, Castelo R, Guinney J. GSVA: gene set variation analysis for microarray and RNA-seq data. BMC Bioinformatics. 2013;14:7.

33. Sun W, Wang L, Zhao D, Wang P, Li Y, Wang S. Four Circulating Long NonCoding RNAs Act as Biomarkers for Predicting Cervical Cancer. Gynecol Obstet Invest. 2018:83(6):533-9.

34. Shen L, Yu H, Liu M, Wei D, Liu W, Li C, et al. A ten-long non-coding RNA signature for predicting prognosis of patients with cervical cancer. OncoTargets and therapy. 2018;11:6317-26.

35. Mao Y, Dong L, Zheng Y, Dong J, Li X. Prediction of Recurrence in Cervical Cancer Using a Nine-IncRNA Signature. Front Genet. 2019;10:284.

36. Luo W, Wang M, Liu J, Cui X, Wang H. Identification of a six IncRNAs signature as novel diagnostic biomarkers for cervical cancer. J Cell Physiol. 2020;235(2):993-1000

37. Lee JY, Lee HS, Kang NW, Lee SY, Kim DH, Kim S, et al. Blood component ridable and CD44 receptor targetable nanoparticles based on a maleimide-functionalized chondroitin sulfate derivative. Carbohyd Polym. 2020;230:115568.

38. Woo HG, Choi JH, Yoon S, Jee BA, Cho EJ, Lee JH, et al. Integrative analysis of genomic and epigenomic regulation of the transcriptome in liver cancer. Nat Commun. 2017:8(1):839.
39. Zammataro L, Lopez S, Bellone S, Pettinella F, Bonazzoli E, Perrone E, et al. Whole-exome sequencing of cervical carcinomas identifies activating ERBB2 and PIK3CA mutations as targets for combination therapy. Proc Natl Acad Sci U S A. 2019;116(45):22730-6.

40. Xu D, Liu S, Zhang L, Song L. MiR-211 inhibits invasion and epithelialto-mesenchymal transition (EMT) of cervical cancer cells via targeting MUC4. Biochem Biophys Res Commun. 2017;485(2):556-62.

41. Despierre E, Moisse M, Yesilyurt B, Sehouli J, Braicu I, Mahner S, et al. Somatic copy number alterations predict response to platinum therapy in epithelial ovarian cancer. Gynecol Oncol. 2014;135(3):415-22.

42. Wang C, Zou H, Chen A, Yang H, Yu X, Yu X, et al. C-Myc-activated long non-coding RNA PVT1 enhances the proliferation of cervical cancer cells by sponging miR-486-3p. J Biochem. 2020.

\section{Publisher's Note}

Springer Nature remains neutral with regard to jurisdictional claims in published maps and institutional affiliations.
Ready to submit your research? Choose BMC and benefit from:

- fast, convenient online submission

- thorough peer review by experienced researchers in your field

- rapid publication on acceptance

- support for research data, including large and complex data types

- gold Open Access which fosters wider collaboration and increased citations

- maximum visibility for your research: over 100M website views per year

At BMC, research is always in progress.

Learn more biomedcentral.com/submissions 